\title{
ORGANIZING CONMITTEE
}

W.N. Brouw (Netherlands)

E. Blum (France)

M.H. Cohen (U.S.A.)

T.W. Cole (Australia)

R.D. Ekers (Netherlands)

E.B. Fomalont (U.S.A.)

M. Ishiguro (Japan)

G.G. Pooley (U.K.)
LOCAL

E. Raimond (Netherlands)

J.P. Hamaker (Netherlands)

R.H. Harten (Netherlands)

R.S. 1e Poole (Netherlands)

U.J. Schwarz (Netherlands)

EDITOR

C. van Schooneveld (Netherlands) 